\title{
Study on Spatial Distribution Characteristics of Intra-city Economic Sector Value-added Growth Rates in Henan Province
}

\author{
Kaiguang Zhang ${ }^{1, a^{*}}$, Mingting $\mathrm{Ba}^{1, b}$, Yanmin Sun ${ }^{1, a}$, Hongling Meng ${ }^{1,2, \mathrm{c}}$ \\ ${ }^{1}$ Institute of 3S Technology, Zhengzhou Normal University, Zhengzhou ,China \\ 2 Institute of Curriculum and Instruction, East China Normal University, Shanghai, China \\ azzgis@sina.com, bbmt1234@126.com, chnmhl@126.com
} Keywords: Henan province; sector value-added growth rate; spatial statistical analysis; cluster
analysis; spatial correlation; spatial difference degree.

\begin{abstract}
Using city region as the study unit, this paper utilizes spatial statistical analysis methods to analyze the spatial distribution characteristics, spatial correlations, as well as the difference degrees of sector value-added growth rates for all cities of Henan province in 2011-2014. The results show that the sector development policies are relatively close among the cities, there is a polarization feature of sector value-added growth rates in sectors, the sector value-added growth rates of agriculture and industry have significant negative correlations among the cities, and a clear trend of middle high and side low in the province.
\end{abstract}

\section{Introduction}

City regional economy, which is city-level administrative divisions geographic unit, is an important component of the regional economy with not only some form of geographical features but fully functional economic characteristics[1,2]. It is extremely significant to scientifically evaluate city regional economic development by sector, to research the distribution of the main driving forces of economic growth, to analyze and compare the differences between the cities, for revealing the weakness and development directions of different regional economic growth, promoting regional economic cooperation, health and sustainable development, as well as comprehensively improving the regional economic development level $[3,4]$.

The city regional economic development is influenced by spatial location and natural resources distribution, has a certain geographical feature, meanwhile one city economic growth rate is closely related to the economic development of the cities around, the spatial distribution of the growth rates has some spatial correlation characteristics. Therefore, for studying the problem of regional economic sector growth rate difference, we need to add geographical factors to the study in the whole process[5], the addition of spatial relations makes the results more comprehensive and scientific [6].

Henan province, located in the core nodes on the Belt and Road Initiative, is the main body of Central Plains Economic Region, and consists of 18 cities. By 2014, there are double-digit economic growth rates for 12 consecutive years. However, the relative large regional economic difference and the overall serious unequal economic development have led to a serious negative impact on the national economy, regional economic and social development[1,2,7].

This paper utilizes the spatial cluster analysis and spatial correlation analysis methods to analyze the value-added growth rates of the main sectors of the national economy from 2010 to 2014 for the cities in Henan province, to study the spatial distribution characteristics and the spatial correlations of sector value-added growth rates, and understand the regional economic growth spatial pattern for the entire province, for unearthing the profound reasons and formulating the corresponding countermeasures to maintain the province's economic equilibrium, rapid and sustainable development supply a scientific basis. 


\section{Data and Processing}

Data. On basis of comprehensiveness, comparability, representativeness and operability principles, references of national economic industrial classification criteria [8], the indicators of this study include agriculture $\left(x_{1}\right)$, industry $\left(x_{2}\right)$, construction $\left(x_{3}\right)$, transportation, storage and postal services $\left(x_{4}\right)$, information transmission, software and information technology services $\left(x_{5}\right)$, wholesale and retail, accommodation and catering industry $\left(x_{6}\right)$, etc.. All data come from Statistical Yearbook of Henan (2010 to 2014), spatial map comes from the space map database of Digital Henan.

Data Processing. First of all, the sector constant price matrices are derived from the sector value-added data of all cities in accordance with constant 2010 prices. Secondly, standardize the matrices by the formal standardization method for each sector of one city. Thirdly, calculate annual value-added growth rate matrices using adjacent annual value-added data. Fourthly, carry out principal component analysis, under the confidence level (90\%) to extract principal component of sector value-added growth rate, calculate the corresponding component score coefficients and latent root, then define the value-added growth rate vectors $V=\left(v_{1}, v_{2}, v_{3}, v_{4}, v_{5}, v_{6}\right)$ for each city as the following:

$$
V=\sum_{m=1}^{M} \sqrt{\lambda_{m}} P_{m}
$$

Where, $M$ is as the number of principal component extracted, $\lambda_{m}$ and $P_{m}$ are respectively the corresponding latent root and component score coefficient vector. Calculation shows that the information extraction yield is over $99.9 \%$ for all cities when extracting 3 principal components, the results are shown in Table1.

Table 1 The sector value-added growth rates in Henan province

\begin{tabular}{|l|c|l|l|l|l|l|l|l|l|l|l|l|l|}
\hline City & $x_{1}$ & $x_{2}$ & $x_{3}$ & $x_{4}$ & $x_{5}$ & $x_{6}$ & City & $x_{1}$ & $x_{2}$ & $x_{3}$ & $x_{4}$ & $x_{5}$ & $x_{6}$ \\
\hline Zhengzhou & 0.56 & 1.42 & 0.38 & 0.81 & 0.99 & 1.38 & Xuchang & 0.74 & 0.56 & 1.60 & -0.46 & -0.82 & 0.02 \\
\hline Kaifeng & 0.92 & 1.33 & -0.68 & 1.14 & 1.24 & 0.35 & Luohe & 0.88 & 1.29 & 1.31 & -0.23 & 0.84 & 1.03 \\
\hline Luoyang & -0.57 & 0.56 & 0.31 & 0.96 & 1.62 & 1.31 & Sanmenxia & 1.23 & 1.16 & 1.44 & -0.39 & 0.95 & 0.01 \\
\hline Pingdingshan & 1.46 & 1.08 & 0.53 & 1.29 & 0.87 & -0.18 & Nanyang & 0.80 & 0.36 & 0.99 & 0.61 & 1.73 & 0.95 \\
\hline Anyang & 0.34 & 1.13 & 1.34 & 0.66 & 1.08 & 1.09 & Shangqiu & 1.19 & 1.07 & 1.47 & 0.98 & -0.39 & 0.44 \\
\hline Hebi & 1.66 & 0.63 & 0.97 & 0.37 & 0.71 & 1.12 & Xinyang & 0.23 & 1.44 & 0.73 & 1.30 & 1.21 & 0.45 \\
\hline Xinxiang & 1.08 & 1.06 & 0.71 & -1.47 & 0.82 & 0.60 & Zhoukou & 0.88 & 0.71 & 0.95 & 1.04 & 0.93 & 1.37 \\
\hline Jiaozuo & 1.19 & 0.81 & 1.17 & 0.31 & -0.26 & 1.55 & Zhumadian & 1.43 & 0.93 & 0.76 & 1.37 & 0.31 & 0.72 \\
\hline Puyang & 0.93 & 0.63 & 1.44 & 0.50 & 1.12 & 1.08 & Jiyuan & 1.43 & 0.93 & 0.76 & 1.37 & 0.31 & 0.72 \\
\hline
\end{tabular}

\section{Research Methods}

The research constructs a spatial weights matrix according to the spatial relationship of 18 cities, and uses the cluster analysis to analyze the distribution of city regional economic sector value-added growth rates, then uses spatial correlation analysis to study their spatial correlation characteristics.

Spatial weight matrix. Spatial weight matrix is a binary symmetric matrix describing the spatial adjacent relationship. On topology, two regions have a common edge set the value as 1 , otherwise as 0 .

K-mean cluster analysis. K-mean cluster analysis is one of the effective spatial clustering methods. The analysis steps are as the following: (1) define the distance between samples and the cluster criterion function, according to the prior knowledge to determine the classification number and the initial cluster center; (2) assign all remaining samples to one of the centers according to the principle of minimum distance, and then calculate the new cluster centers and the criterion function, (3) repeat step (2) until the criterion function is convergence or its change fall in the tolerance interval [9]. 
The global spatial auto-correlation. Global spatial auto-correlation uses Moran I index to show the spatial difference and spatial similarity of the adjacent region data which come form a certain regular probability distribution. The test statistic, standardized $\mathrm{Z}$ value, represents the degree of correlation between the observed values of all regions, if the corresponding significance level is smaller than the lowest significant level (general 0.05 set before), the sample data assume come from a random distribution, there are no spatial correlations. Under the significance level, positive and negative $\mathrm{Z}$ respectively stand for aggregation degree and dispersion degree of observations [10-12].

The local spatial auto-correlation. Local spatial auto-correlation using the Local Moran I index to show the space-related model on different spatial locations, which describes the degree of aggregation and the degree of difference for one region which have some significant similarity with surrounding regions[10-12].

Moran scatter plot. Moran scatter plot is a two-dimensional visualization diagram to describe the local space stability for one region, the horizontal axis represents sample variable, the vertical axis is the spatial weighted average of adjacent region samples, and four quadrants respectively represent high-high (HH), low-high (LH), low-low (LL) and high-low (HL) aggregation [10-12].

\section{The spatial Analysis of Sector Value-added Growth Rates in Henan Province}

The Cluster analysis of sector value-added growth rates in Henan province. In the cluster analysis of sector value-added growth rates, Euclidean distance is used as the distance between two samples, the sum of deviations squares is taken as criterion function. According to the prior study on the distribution characteristics of the regional economy of the province , 3 is adopted as the number of clusters, and Shangqiu, Luoyang and Jiaozuo, which are respectively the important agricultural base, the industrial base and the strong tourist base, are selected as the initial cluster centers. The final cluster centers and cluster results are shown in Table2 and Fig.1.

The cluster results show that the economic growth in the province could be divided into 3 classes, their spatial distribution is showed on the Fig.1.

The first class is construction driving type, represented by Xinxiang, composed of Hebi and Sanmenxia, the economic growth mainly successively come from construction, industry, agriculture, transportation, and so on. Storage and postal service are lagged behind. The second class, represented by Zhumadian, including Pingdingshan, Hebi, Jiaozuo, Puyang, Xuchang, Shangqiu, Zhumadian and Jiyuan, etc., is driven by agriculture, the major sector economic growth points are agriculture and construction, the economic growth of other sectors is a relative balanced. The third group is a new industry driving type, the economic growth mainly comes from the emerging industry as information transmission, software and information technology services, traditional industry, wholesale and retail, accommodation and catering industry, represented by Zhengzhou, including Luoyang, Kaifeng, Anyang, Nanyang , Xinyang city Zhoukou city, etc..

Table 2 The final cluster centers

\begin{tabular}{|r|r|l|l|l|l|l|}
\hline Class & \multicolumn{1}{|l|}{$x_{1}$} & \multicolumn{1}{l|}{$x_{2}$} & \multicolumn{1}{l|}{$x_{3}$} & \multicolumn{1}{l|}{$x_{4}$} & \multicolumn{1}{l|}{$x_{5}$} \\
\hline 1 & 1.07 & 1.17 & 1.15 & -0.70 & 0.87 & 0.55 \\
\hline 2 & 1.25 & 0.83 & 1.09 & 0.72 & 0.23 & 0.69 \\
\hline 3 & 0.45 & 0.99 & 0.57 & 0.93 & 1.26 & 0.99 \\
\hline
\end{tabular}

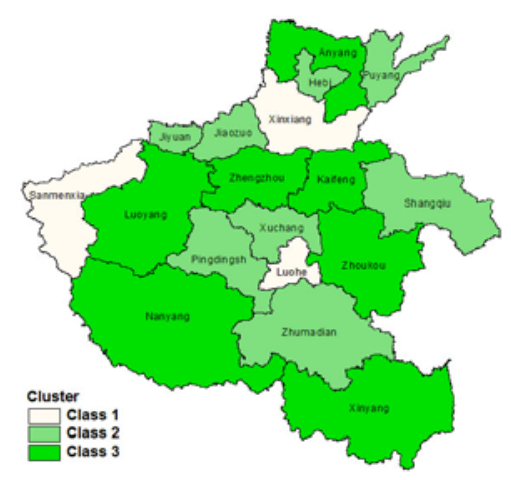

Fig.1 The cluster analysis of sector value-added growth rates by city

The global spatial auto-correlation analysis. Using the spatial auto-correlation analysis on the 6 indicators of the city regional economic sector value-added growth rates, the standardized Moran I index, $\mathrm{Z}$ and $\mathrm{P}$ values are shown in Table 3. There are some obvious auto-correlation in the traditional sector as agriculture and industry, under the significant level (0.05), the negative Moran Is show that there are some negative auto-correlation in the two sectors among the cities, which show the characteristics of low-high and high-low aggregation, in other words, the influences between the 
adjacent cities are very little, the city regional economic developments are relatively close. Although there is some certain auto-correlation in the other four sectors, but the degrees of auto-correlation are very weak, the contributions of the sector economic growths to the overall economic growth are relatively balanced.

Table 3 The Global spatial auto-correlation analysis of sector value-added growth rates by city

\begin{tabular}{|l|r|r|r|r|r|r|}
\hline & \multicolumn{1}{|c|}{$x_{1}$} & \multicolumn{1}{l}{$x_{2}$} & \multicolumn{1}{l}{$x_{3}$} & \multicolumn{1}{l|}{$x_{4}$} & \multicolumn{1}{l}{$x_{5}$} & \multicolumn{1}{l}{$x_{6}$} \\
\hline Moran I & -0.3220 & -0.3382 & -0.1483 & -0.0065 & -0.0964 & -0.1480 \\
\hline Z value & -1.9950 & -2.0251 & -0.7549 & 0.3594 & -0.2531 & -0.5655 \\
\hline a value & 0.0032 & 0.0045 & 0.2450 & 0.3340 & 0.4313 & 0.3073 \\
\hline
\end{tabular}
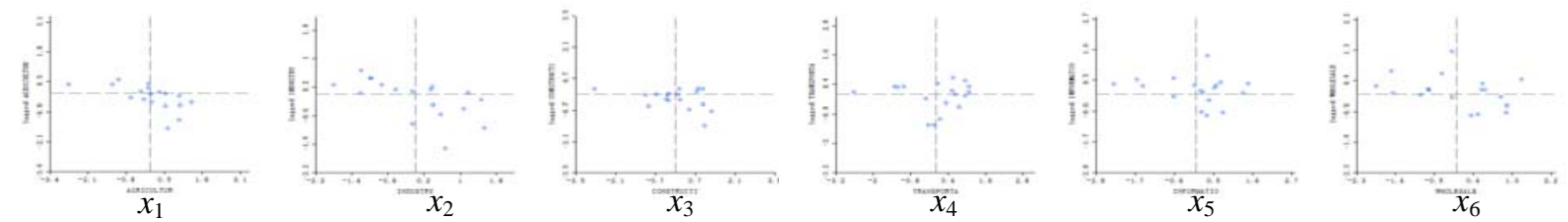

Fig.2 The Moran scatter plots of cluster analysis of sector value-added growth rates
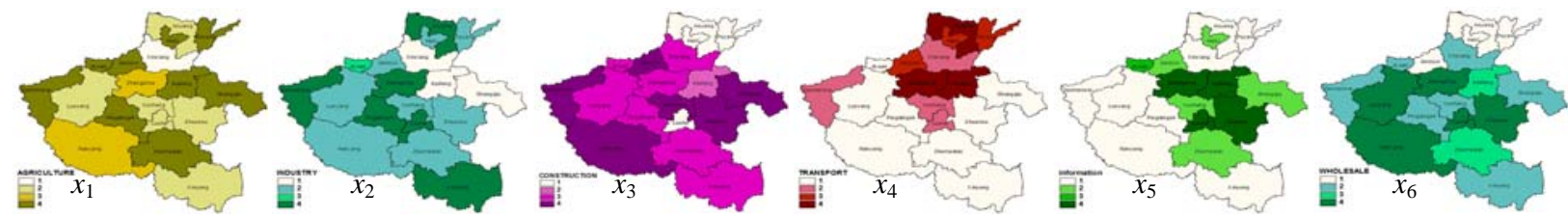

Fig.3 The spatial auto-correlation distributions of sector value-added growth rates

Moran scatter plot. Fig.2 and Fig.3 respectively are the Moran scatter plots and spatial distributions of the sector value-added growth rates, which show that most of cities, in the sector of transportation, storage and postal services, are located in the first or the third quadrant, means there are weekly positive correlation. About other 5 sectors, most of cities are located in the second and fourth quadrant, means there are some negative correlation.

The agricultural value-added growth rates among cities are very unbalanced in the spatial distribution, faster growing regions are mainly concentrated around Zhengzhou and Luoyang, and along the Lianhuo highway, while the sector value-added growth rates of main industrial base, as Zhengzhou and Luoyang, are relative slowly. The industrial value-added growth rates show an uneven distribution features, faster growth regions are mainly concentrated in the middle of the province and the cities located on the periphery of the province, as Sanmenxia, Xinyang and Anyang where have weekly industrial bases. Construction presents strong level of low-low and high-low aggregation features, sector value-added growth rates exhibit low-central and high-side trend. Transportation, storage and postal industry has strongest positive correlation in the 6 sectors, the sector value-added growth rates in the central and northern regions are slower than other regions. Information transmission, software and information technology services renders the characteristics of high-low aggregation around low-high aggregation, the fastest growing regions are around here. There are big correlation differences in the wholesale, retail, accommodation and catering, the fastest growing regions are gathered in the mid-western and southern of the province.

Local spatial auto-correlation analysis. Only agricultural and industrial value-added growth rates exhibit an obvious spatial auto-correlation, under the significant level (0.05), according to the local spatial auto-correlation analysis (see Fig.4).

On view of the spatial location distribution, form Fig. 4 and Table1, there is no high-high and low-low aggregation regions, The aggregation regions of low-high and high-low are located on the periphery of the province, where have relatively complex terrain, and low sector economic development level. The agricultural and industrial value-added growth rates of Sanmenxia affected by

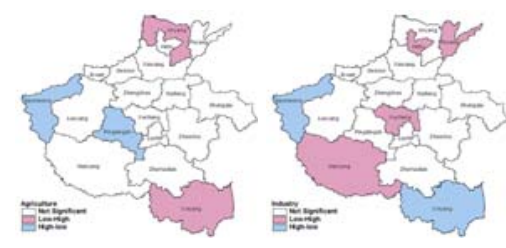

Fig.4 The local Moran I plot of agriculture and industry 
Luoyang, are high-low aggregation. The agricultural value-added growth rate of Xinyang is low-high aggregation, while the industrial value-added growth rate is high-low aggregation, which indicates there is a relatively uncoordinated agricultural and industrial development in the city.

On view of the spatial interaction, form Fig.4 and Tab.1, there are discrete distribution characteristics of low-high and high-low aggregation. The mutual influence on the economic development is poor, and the city regional economic development is relatively close. The phenomenon is more obvious in the 3 cities on the northern of the province, the agricultural value-added growth rate of Anyang is low-high aggregation, while the industrial value-added growth rates of adjacent cities, as Hebi and Puyang, are low-high aggregation.

\section{Conclusions and Recommendations}

The paper utilizes the spatial statistical analysis method to study the spatial distribution characteristics and the spatial correlations of city regional economic sector value-added growth rates in Henan province. The results show that: (1)the city regional economic growth could be mainly divided into three classes as the emerging industry driving type, the agriculture driving type and construction driving type. (2)There are significant negative correlations in the agriculture and industry value-added growth rates, which are very weak in the other four sectors, indicating that the sector development policies are relatively close among the cities, the mutual influence is poor.(3)There are a significant difference in the agriculture and industry value-added growth rates, which render the characteristic of the high-central and low-side aggregating at the province capital.

In short, the economic development of Henan province should be continued to implement a balanced development strategy, break the regional restrictions, expand the mutual influence of regional economy, increase the intensity of macroeconomic regulation and control, in order to fully guarantee the balanced, healthy and sustainable rapid development of economy, and comprehensively improve the provincial economic development level, meanwhile speed up the characteristics construction of key regions, enhance their polarization functions, and promote regional coordinated development.

\section{References}

[1] W. F. Chen, W. L. Zhao, D. Y. Meng, Evolvement of temporal-spatial pattern of economic development in Central Plain Economic Zone, Economic Geography. 10(2011), p. 1585-1591.

[2] Y. J. Qi, Y. J. Yang, J. Feng, China's economic development stage and its patio-temporal evolution: A prefectural-level analysis, Geographical Science. 4(2013), p. 517-531.

[3] L. M. Hu, C. H. Miao, J. J. Qiao, A study on the divergence and temperal-spatial structure of regional economic development in Henan Province, Progress in Geography, 3(2002) , p. 268-274.

[4] F. Q. Song, Z. L. Zheng, D. X. Feng, Charactor and optimization method of spatial agglomeration of population and economy in Henan province, Areal Research and Development, 6(2013), p. 116-119.

[5] J. Fan, The strategy of major function oriented zoning and the optimization of territorial development patterns, Bulletin of Chinese Academy of Sciences, 2(2013) , p. 193-206.

[6] Y. C. Gong, J. Feng, J. Luo, Research on time and space charactorixtics of the three nre-type coordinated development level of the Central Plains Economic Region, Areal Research and Development, 2013, 32 (3), p. 158-171.

[7] Q. X. Ning, T. Zhang, An Chunhua. Accessing on coordination degree of regional development in Henan Province, Areal Research and Development, 6(2014), p. 33-38.

[8] P. M. Pan, Research on the classification standards of the national economy industry, Statistical Theory and Practice, 6(2012), p. 16-18. 
[9] M. W. Wang, H. Ye, J. L. Zuo, Document clustering based on constrained principal component analysis, Pattern Recognition and Artificial Intelligence, 3(2013), p. 270-275.

[10] k. Liu, Y. M. Shen, Study on industrial association effect of Henan province, Areal Research and Development, 1(2013), p. 29-34.

[11] Y. J. Qi, Y. Yang, F. J. Jin, China's economic development stage and its patio-temporal evolution:A prefectural-level analysis, Acta Geographica Sinica, 4(2013), p. 517-531.

[12] L. J. Zhang, Y. C. Qin, J. P. Zhang. County green GDP accounting based on EMB-MFA method and its spatial differentiantion: a case of Henan province, Journal of Natural Resources, 3(2013), p. 504-515. 